\title{
Angular Momentum in Rotating Superfluid Droplets
}

\author{
Sean M. O. O’Connellø, ${ }^{1}$ Rico Mayro P. Tanyag $\odot,{ }^{1,2}$ Deepak Verma, ${ }^{1}$ Charles Bernando $\odot,{ }^{3,4}$ Weiwu Pang, ${ }^{5}$ \\ Camila Bacellar, ${ }^{6,7}$ Catherine A. Saladrigas, ${ }^{6,7}$ Johannes Mahl $\odot,{ }^{6,8}$ Benjamin W. Toulson, ${ }^{6}$ Yoshiaki Kumagai $\odot,{ }^{9}$ \\ Peter Walter, ${ }^{10}$ Francesco Ancilotto $\odot,{ }^{11,12, *}$ Manuel Barranco $\odot,{ }^{13,14,15}$ Marti Pi®, ${ }^{13,14}$ Christoph Bostedt, $, 916,17,18$ \\ Oliver Gessner $\oplus^{6, \dagger}$ and Andrey F. Vilesov $\oplus^{1,3, *}$ \\ ${ }^{1}$ Department of Chemistry, University of Southern California, Los Angeles, California 90089, USA \\ ${ }^{2}$ Technische Universität Berlin, Institut für Optik und Atomare Physik, 10623 Berlin, Germany \\ ${ }^{3}$ Department of Physics and Astronomy, University of Southern California, Los Angeles, California 90089, USA \\ ${ }^{4}$ OVO (PT. Visionet Internasional), Lippo Kuningan 20th floor, Jalan HR Rasuna Said No. B-12, Setiabudi, Jakarta 12940, Indonesia \\ ${ }^{5}$ Department of Computer Science, University of Southern California, Los Angeles, California 90089, USA \\ ${ }^{6}$ Chemical Sciences Division, Lawrence Berkeley National Laboratory, Berkeley, California 94720, USA \\ ${ }^{7}$ Department of Chemistry, University of California Berkeley, Berkeley, California 94720, USA \\ ${ }^{8}$ Department of Physics, University of Hamburg, 22761 Hamburg, Germany \\ ${ }^{9}$ Chemical Sciences and Engineering Division, Argonne National Laboratory, \\ 9700 South Cass Avenue B109, Lemont, Illinois 60439, USA \\ ${ }^{10}$ Linac Coherent Light Source, SLAC National Accelerator Laboratory, Menlo Park, California 94025, USA \\ ${ }^{11}$ Dipartimento di Fisica e Astronomia and CNISM, Università di Padova, 35122 Padova, Italy \\ ${ }^{12}$ CNR-IOM Democritos, 34136 Trieste, Italy \\ ${ }^{13}$ Departament FQA, Universitat de Barcelona, Facultat de Física, 08028 Barcelona, Spain \\ ${ }^{14}$ Institute of Nanoscience and Nanotechnology (IN2UB), Universitat de Barcelona, 08028 Barcelona, Spain \\ ${ }^{15}$ Laboratoire des Collisions, Agrégats et Réactivité, IRSAMC, Université Toulouse 3, F-31062 Toulouse, France \\ ${ }^{16}$ Department of Physics and Astronomy, Northwestern University, 2145 Sheridan Road, Evanston, Illinois 60208, USA \\ ${ }^{17}$ Paul Scherrer Institut, Forschungsstrasse 111, 5232 Villigen-PSI, Switzerland \\ ${ }^{18}$ LUXS Laboratory for Ultrafast X-ray Sciences, Institute of Chemical Sciences and Engineering, \\ École Polytechnique Fédérale de Lausanne (EPFL), CH-1015 Lausanne, Switzerland
}

(Received 12 September 2019; accepted 16 April 2020; published 26 May 2020)

\begin{abstract}
The angular momentum of rotating superfluid droplets originates from quantized vortices and capillary waves, the interplay between which remains to be uncovered. Here, the rotation of isolated submicrometer superfluid ${ }^{4} \mathrm{He}$ droplets is studied by ultrafast $\mathrm{x}$-ray diffraction using a free electron laser. The diffraction patterns provide simultaneous access to the morphology of the droplets and the vortex arrays they host. In capsule-shaped droplets, vortices form a distorted triangular lattice, whereas they arrange along elliptical contours in ellipsoidal droplets. The combined action of vortices and capillary waves results in droplet shapes close to those of classical droplets rotating with the same angular velocity. The findings are corroborated by density functional theory calculations describing the velocity fields and shape deformations of a rotating superfluid cylinder.
\end{abstract}

DOI: $10.1103 /$ PhysRevLett.124.215301

A quiescent droplet is spherical, whereas it experiences pronounced shape deformations when set into rotation. Classical viscous droplets execute rigid body rotation (RBR), the kinematics of which are well understood [1-4]. In contrast, a superfluid droplet is described by a quantum mechanical wave function [5,6]. At low temperatures, the angular momentum of a superfluid droplet may be stored in quantized vortices or, as much less frequently discussed, in capillary waves. Both phenomena are fundamentally different from RBR, which poses intriguing questions regarding their relative contributions when comparing classical and quantum mechanical kinematics. Experimental studies of rotation in single, free superfluid ${ }^{4} \mathrm{He}$ droplets have recently become feasible with the advent of x-ray free electron lasers (XFELs) and intense extreme ultraviolet (XUV) sources [7-10]. Ultrafast diffraction experiments have established the existence of oblate axisymmetric and triaxial prolate superfluid droplets with shapes that are found in classical droplets [1-4]. However, previous experiments could not independently probe the angular momentum and angular velocity of rotating droplets, which is imperative for understanding their laws of motion. We address this challenge by simultaneously determining the angular momentum $L$, the angular velocity $\omega$, and the corresponding shapes of the rotating droplets via imaging of both the shapes of ${ }^{4} \mathrm{He}$ droplets and the vortex configurations they contain.

The shapes of classical droplets undergoing RBR follow a universal stability diagram in terms of reduced angular 


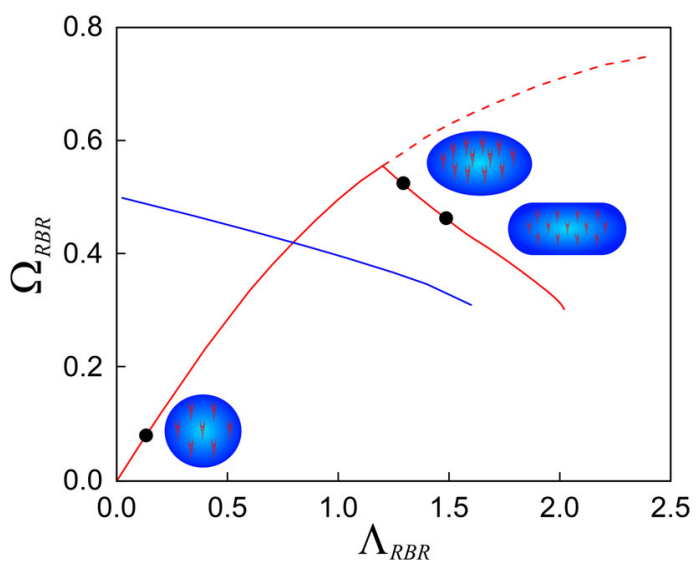

FIG. 1. Stability diagram for classical droplets executing RBR. Red solid traces indicate stable shapes for specific combinations of reduced angular velocity $\Omega_{\mathrm{RBR}}$ and reduced angular momentum $\Lambda_{\mathrm{RBR}}[3,4]$. The left branch corresponds to oblate axisymmetric shapes, the right branch to prolate shapes. The unstable portion of the branch is indicated by a dashed curve. The blue curve corresponds to droplets rotating solely through capillary wave motion [11]. Black circles mark the locations of classical droplets with the same aspect ratios as the superfluid droplets studied in this work.

momentum $\Lambda_{\mathrm{RBR}}$ and reduced angular velocity $\Omega_{\mathrm{RBR}}$, as shown in Fig. 1 [1-4]. The reduced quantities are linked to the angular momentum $L_{\mathrm{RBR}}$ and angular velocity $\omega_{\mathrm{RBR}}$ according to

$$
\begin{gathered}
\Lambda_{\mathrm{RBR}}=\frac{1}{\sqrt{8 \sigma \rho R^{7}}} L_{\mathrm{RBR}} \\
\Omega_{\mathrm{RBR}}=\sqrt{\frac{\rho R^{3}}{8 \sigma}} \omega_{\mathrm{RBR}} .
\end{gathered}
$$

Here, $\sigma$ is the surface tension of the liquid; $\rho$ its mass density; and $R$ the radius of a spherical droplet with the same volume as the deformed droplet. With increasing $\Lambda_{\mathrm{RBR}}$, the droplets become more oblate. At $\Lambda_{\mathrm{RBR}}>1.2$, however, they assume prolate shapes such as pseudotriaxial ellipsoids, capsules, and dumbbells.

Rotation of prolate superfluid droplets can be accomplished by capillary waves, which decay rapidly in classical droplets due to viscosity-induced energy dissipation but may be sustained indefinitely in superfluids. Capillary waves in levitating helium droplets have been investigated experimentally and theoretically $[12,13]$. Traveling capillary waves give rise to a new branch in the stability diagram as shown by the blue line in Fig. 1 [11]. Prolate superfluid droplets likely contain both capillary waves and vortices, whose combined action determines the droplet shape. The arrangement of vortices in rotating, nonaxisymmetric systems represents a general problem that has been discussed theoretically in connection with liquid helium $[11,14]$ and dilute Bose-Einstein condensates (BECs)
[15-18]. The observation, however, of vortices in rotating systems with large asymmetries remains challenging [19].

We posit that in axisymmetric, oblate superfluid droplets, angular momentum originates solely from quantized vortices that arrange in a triangular lattice $[7,20]$ similar to those previously observed in BECs [5,6,21]. In contrast, the angular momentum in prolate superfluid droplets has significant contributions from vortices and capillary waves. We find that in capsule-shaped droplets, vortices form a triangular lattice, whereas they are arranged along elliptical contours in ellipsoidal droplets. The combined action of the vortex lattice and the capillary waves yields droplet shapes close to those in isochoric classical droplets rotating at the same angular velocity.

Experiments were performed using the LAMP end station at the Atomic, Molecular, and Optical (AMO) beam line of the Linac Coherent Light Source (LCLS) XFEL $[22,23]$. Sub-micrometer sized helium droplets containing $N_{\mathrm{He}}=10^{9}-10^{11}$ atoms are produced via fragmentation of liquid ${ }^{4} \mathrm{He}$ expanded into vacuum through a $5 \mu \mathrm{m}$ nozzle at a temperature of $4.5 \mathrm{~K}$ and a backing pressure of $20 \mathrm{bar}$ $[7,24,25]$. The droplets evaporatively cool in vacuum to $T=0.37 \mathrm{~K}[25,26]$, becoming superfluid at $T \approx 2.17 \mathrm{~K}$. To visualize vortices, the droplets are doped with Xe atoms, which cluster along the vortex cores [7,27-30]. The XFEL is operated at $120 \mathrm{~Hz}$ with a photon energy of $1.5 \mathrm{keV}$ $(\lambda=0.826 \mathrm{~nm})$ and a pulse width of $<180 \mathrm{fs}$. Small angle diffraction images are recorded on a $p-n$ charge-coupled device detector centered along the XFEL beam axis. The patterns predominantly contain column density information of the scattering object in the direction perpendicular to the detector plane.

Diffraction patterns from doped droplets with slightly oblate axisymmetric, triaxial pseudoellipsoidal, and capsule shapes are presented in a logarithmic color scale in Figs. 2(a1), 2(b1), and 2(c1), respectively. Figures 2(a2)2(c2) show the corresponding column densities of $\mathrm{He}$ (blue) and Xe (red and yellow) obtained from the diffraction patterns using the droplet coherent diffractive imaging (DCDI) algorithm [28,31], see Supplemental Material for details [32]. The diffraction pattern in Fig. 2(a1) exhibits a circular ring structure close to the center and a speckled pattern in the outer region, which is due to scattering off the He droplet and embedded Xe clusters, respectively. The projection of the droplet contour onto the detector plane is circular with a radius of $308 \pm 6 \mathrm{~nm}$ [7,27,31]. The droplet in Fig. 2(a2) contains 12 vortex cores that are aligned along the droplet rotational axis and extend between opposite surface points. The filaments appear shorter than the droplet diameter, indicating that the rotational axis is tilted with respect to the $\mathrm{x}$-ray beam by $\sim 0.3 \mathrm{rad}$.

Figures 2(b1) and 2(c1) exhibit elongated diffraction contours, with Fig. 2(c1) also featuring a pronounced streak and a regular Bragg pattern. Most noticeably, two parallel lines of four Bragg spots each are arranged symmetrically 


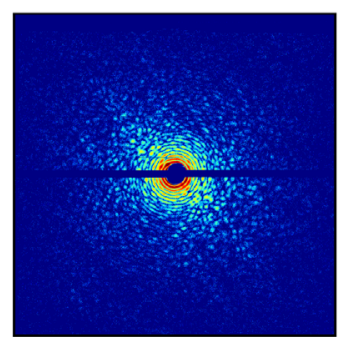

(a1)

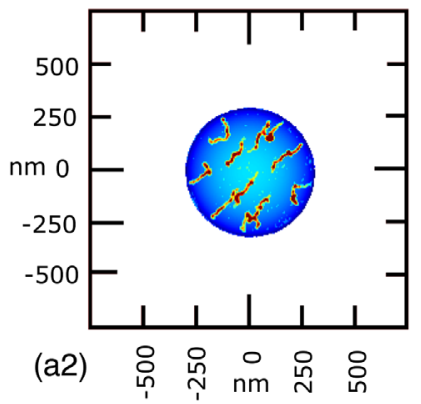

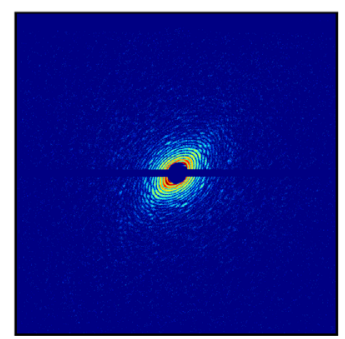

(b1)

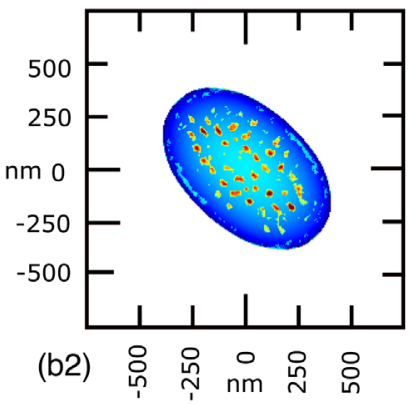

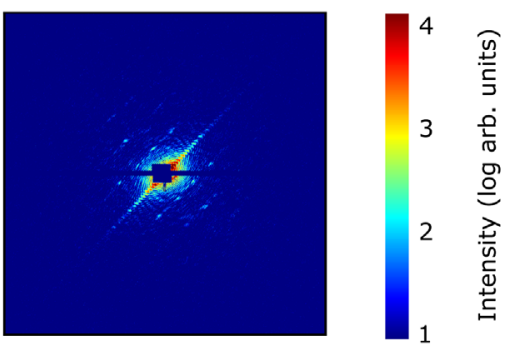

(c1)

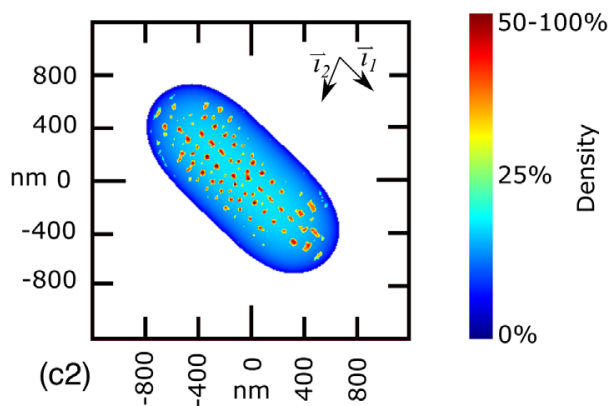

FIG. 2. Diffraction patterns from Xe-doped droplets with various shapes: (a1) axisymmetric, nearly spherical, (b1) triaxial pseudoellipsoidal, and (c1) capsule shaped. The horizontal stripe in (a1)-(c1) results from the gap between the upper and lower detector panels. Panels (a2)-(c2) show column densities retrieved via the DCDI algorithm. The basis vectors of the vortex lattice in (c2) are shown in the upper right corner of the panel.

with respect to the streak. We assign the Bragg spots to $\mathrm{x}$-ray scattering off a lattice of vortex-bound Xe clusters [7]. Figure 2(c2) shows that the droplet contains $\sim 50$ vortices arranged in four rows parallel to the droplet's long axis. The Xe clusters in Fig. 2(c2) appear as compact spots, although they are likely filaments as in Fig. 2(a2). Therefore, the filaments must be aligned perpendicular to the detector plane and the droplet's angular momentum along the $\mathrm{x}$-ray beam.

The droplet in Fig. 2(b2) has a pseudoellipsoidal shape and the pattern of vortices within resemble the droplet's outer contour. The Xe clusters show some elongation along the long axis, indicating a tilt of $\leq 0.15 \mathrm{rad}$ around the droplet's intermediate axis. Since the angular momentum of the droplets in panels 2(b) and 2(c) is aligned approximately along the x-ray beam, the droplet contours yield the actual values of the major and intermediate half axes, $a$ and $b$, as well as the droplet aspect ratio: $\mathscr{A}=a / b$. The minor half-axis $c$, parallel to the rotational axis, cannot be directly determined.

Figures 2(a2), 2(b2), and 2(c2) showcase the evolution of vortex arrangements with changing droplet shapes. Because of the small abundance of fast rotating droplets crossing the x-ray beam focus with proper orientation, only the two diffraction patterns shown in Figs. 2(b1) and 2(c1) were suitable for reliable image phase retrieval. These are considered representative of droplets with similar aspect ratios since any nonequilibrium configurations decay before the droplets reach the interaction point with the imaging x-ray beam [7]. In the spheroidal droplet in
Fig. 2(a2), the vortices are distributed across the entire volume and at regular distances from each other. In Figs. 2(b2) and 2(c2), the vortices only occupy the central part of their respective droplets, leaving $\sim 100$ and $\sim 150 \mathrm{~nm}$ wide boundary regions devoid of vortices, respectively. The vortices in the prolate droplet are arranged in four rows along the droplet's long axis. The vortex lattice is reciprocal to that of the Bragg spots and is defined as

$$
\vec{R}=m_{1} r_{1} \vec{i}_{1}+m_{2} r_{2} \vec{i}_{2},
$$

where $m_{1}$ and $m_{2}$ are integers, $r_{1}=111 \mathrm{~nm}, r_{2}=100 \mathrm{~nm}$, and $\vec{i}_{1}$ and $\vec{i}_{2}$ are unit vectors with $\vec{i}_{1}$ perpendicular to the streak and $\vec{i}_{2}$ oriented at a $67^{\circ}$ angle relative to $\vec{i}_{1}$ as illustrated in Fig. 2(c2). The vortex lattice is triangular, but not equilateral as in the idealized 2D case [5] and as previously observed in axisymmetric rotating He droplets [7] and BECs [5,6,21]. These observations suggest that the droplet shapes and vortex patterns within are closely interrelated.

We compare the angular momentum and angular velocity in superfluid droplets in Figs. 2(b2) and 2(c2) to those of their classical counterparts. From the measured aspect ratios in Figs. 2(b2) and 2(c2), we first obtain the values of the reduced quantities $\Lambda_{\mathrm{RBR}}$ and $\Omega_{\mathrm{RBR}}[3,4,8]$ for the corresponding classical rotating droplets and then calculate $L_{\mathrm{RBR}}$ and $\omega_{\mathrm{RBR}}$ according to Eqs. (1) and (2). The angular momentum due to vortices $L_{\mathrm{VORT}}$ is obtained from the number of vortices $N_{V}$ and their location within the droplet 
[33] as detailed in the Supplemental Material [32]. The angular velocity of the vortex array is obtained from the average vorticity as [34]

$$
\omega_{\mathrm{VORT}}=\frac{n_{v} \kappa}{2},
$$

where $n_{v}$ is the areal number density of vortices and $\kappa=h / m_{4}$ is the quantum of circulation, derived from Planck's constant $h$ and the mass of the ${ }^{4} \mathrm{He}$ atom $m_{4}$.

The angular velocity of the superfluid droplet is given by $\omega_{\mathrm{SF}}=\omega_{\mathrm{VORT}}$ because the vortex array must be stationary with respect to the droplet contour at equilibrium. The angular momentum due to capillary waves $L_{\mathrm{CAP}}$ is obtained as the product of the effective irrotational moment of inertia of the droplets multiplied by $\omega_{\mathrm{SF}}[32,35]$. Contributions of $\mathrm{Xe}$ tracers to the angular momentum are neglected since the total mass of the embedded Xe atoms in Fig. 2 is $~ 100$ times smaller than that of the He droplet and the vortices doped with Xe atoms mostly occupy the central region of the droplet. The impact of doping on vortex kinematics in spherical droplets has been studied previously $[27,38]$. It was concluded that bare and Xe-decorated vortices have similar angular momenta when the vortices are located near the droplet's center as is the case here.

Table I lists the kinematic parameters of the rotating superfluid droplets in Fig. 2. The total angular momentum in superfluid prolate droplets [(b2), (c2)] $L_{\mathrm{SF}}=L_{\mathrm{VORT}}+$ $L_{\mathrm{CAP}}$. The $L_{\mathrm{CAP}} / L_{\mathrm{VORT}}$ ratio decreases with decreasing aspect ratio, $\mathscr{A}$, ultimately meeting the condition $L_{\mathrm{CAP}}=0$ in axisymmetric droplets (a2). The analysis also reveals that, within the accuracy of our estimates, $L_{\mathrm{SF}} \approx L_{\mathrm{RBR}}$ and $\omega_{\mathrm{SF}} \approx$ $\omega_{\mathrm{RBR}}$ for isochoric classical and vortex-hosting superfluid droplets of similar shape. More generally, the results in Table I suggest that rotation of large, vortex-hosting superfluid droplets can be described in terms of a reduced angular velocity and a reduced angular momentum in a similar fashion as the rotational motion of their classical counterparts. Details of the analysis and a comprehensive list of geometric and kinematic parameters are presented in the Supplemental Material [32], which also notes differences between classical and superfluid droplets having the same aspect ratio.

To rationalize the similarities between the shapes of classical and superfluid droplets, we may recall that, for a given $L$, RBR results in the smallest kinetic energy.

TABLE I. Kinematic parameters for the droplets in Fig. 2.

\begin{tabular}{lccc}
\hline \hline Quantity & (a2) & (b2) & $(\mathrm{c} 2)$ \\
\hline$L_{\mathrm{RBR}} /\left(N_{\mathrm{He}} \times \hbar\right)$ & 4.8 & 50 & 73 \\
$\omega_{\mathrm{RBR}}, \mathrm{rad} / \mathrm{s}$ & $2.0 \times 10^{6}$ & $1.24 \times 10^{7}$ & $4.9 \times 10^{6}$ \\
$\omega_{\mathrm{SF}}, \mathrm{rad} / \mathrm{s}$ & $2.0 \times 10^{6}$ & $9.7 \times 10^{6}$ & $4.9 \times 10^{6}$ \\
$L_{\mathrm{VORT}} /\left(N_{\mathrm{He}} \times \hbar\right)$ & 4.8 & 31 & 30 \\
$L_{\mathrm{CAP}} /\left(N_{\mathrm{He}} \times \hbar\right)$ & 0 & 18 & 48 \\
$L_{\mathrm{SF}} /\left(N_{\mathrm{He}} \times \hbar\right)$ & 4.8 & 49 & 78 \\
\hline \hline
\end{tabular}

A vortex array in the central part of a droplet creates a region of liquid that effectively moves as in RBR and minimizes the required capillary engagement. Essentially, the prolate droplet attempts to approach RBR by combining the effect of vortices and capillary waves. It appears that for each $L$, there is an optimal partitioning into $L_{\mathrm{VORT}}$ and $L_{\mathrm{CAP}}$ that minimizes the total energy of the rotating droplet.

To ascertain how the shape of a superfluid droplet changes with the number of vortices at constant angular momentum, we have carried out density functional theory (DFT) calculations $[11,36,37]$. Computationally costly 3D calculations can only be performed for small droplets, which cannot support a large number of vortices [11]. Therefore, we study a freestanding, deformable ${ }^{4} \mathrm{He}$ cylinder, rotating around its symmetry axis. Calculations are performed in the corotating frame [37]. The total angular momentum is fixed at $L_{\mathrm{SF}} / N_{\mathrm{He}}=7.83 \hbar$.

Figure 3 shows the results of the calculations with different numbers of vortices, ranging from $N_{V}=2$ to $N_{V}=11$. The deformation of the cylinder increases for decreasing numbers of vortices, as capillary waves contribute more angular momentum. Note that the energies of the configurations in Fig. 3 are similar; they differ less than $0.01 \mathrm{~K}$ per atom, with the $N_{V}=6$ configuration [(Fig. 3(c)] representing the global minimum. Each panel in Fig. 3 shows the computed $L_{\mathrm{CAP}} / L_{\mathrm{SF}}$ ratio and the cylinder's aspect ratio and $\omega$. In cylinders with relatively small $\mathscr{A}=1.1$ and 1.5 , the vortices are arranged into elliptical patterns, whereas they form elongated arrays for larger aspect ratios, in agreement with the experimental observations in Fig. 2.

Figure 3 illustrates how the effects of vortices and capillary waves confer the appearance of RBR to the motion of the superfluid. Each panel shows the superfluid streamlines, illustrating the different irrotational velocity fields: the streamlines dominated by vortices wrap around the vortex cores, whereas those associated with capillary waves terminate at the surface.

Figure 3(d) shows an elongated droplet containing eight vortices arranged in a pattern reminiscent of a distorted square lattice with an average nearest-neighbor distance of $d=4.7 \mathrm{~nm}$ and an areal vortex density of $1 / d^{2}=4.5 \times 10^{16} \mathrm{~m}^{-2}$. According to Eq. (4), the angular velocity is $\omega=2.26 \times 10^{9} \mathrm{rad} / \mathrm{s}$ in excellent agreement with the DFT result of $\omega=2.23 \times 10^{9} \mathrm{rad} / \mathrm{s}$. Thus, the results of the DFT calculations lend further support for using Eq. (4) to determine $\omega$ of rotating He droplets from the finite vortex arrays they contain.

This combined experimental and theoretical study provides the first direct comparison between the shapes, angular velocities, and angular momenta of rotating classical and superfluid droplets. The results indicate that the equilibrium figures of superfluid droplets hosting vortices may be described in a similar fashion as those of classical, viscous droplets by a series of oblate and 

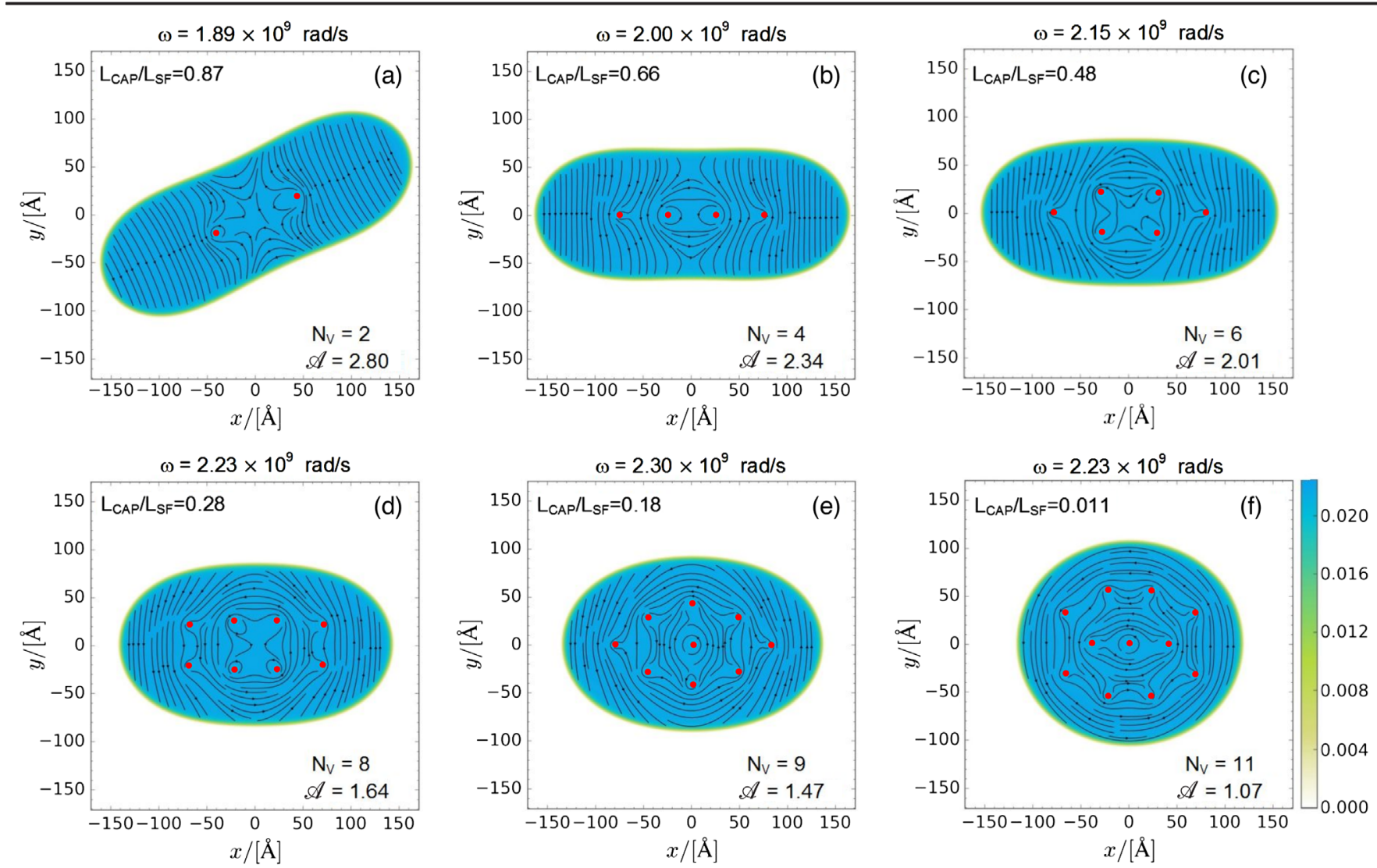

FIG. 3. Calculated equilibrium density profiles of a deformable ${ }^{4} \mathrm{He}$ cylinder rotating around its symmetry axis at fixed $L_{\mathrm{SF}} / N_{\mathrm{He}}=$ $7.83 \hbar$ for different numbers of vortices. Streamlines are shown in black. The positions of the vortex cores are marked by red dots for visualization. The color bar shows the density in units of $\AA^{-3}$.

prolate shapes that evolve along curves of stability as a function of reduced angular momentum $\Lambda$ and reduced angular velocity $\Omega$. In axisymmetric oblate superfluid droplets, all angular momentum is stored in triangular lattices of quantum vortices that extend throughout the entire droplet volume. In prolate droplets, however, capillary waves can contribute a substantial and, for large aspect ratios, even dominant amount of angular momentum. It appears that prolate superfluid droplets approach the classical equilibrium figures through a combination of vortices and capillary motion, whereby the vortex arrangements are restricted to the central region of the droplet.

Future experiments at high-repetition rate ultrafast x-ray light sources will provide larger sample sizes and explore more expansive ranges of $\Lambda, \Omega$, and $R$. More advanced theoretical work is required to study angular velocities and angular momenta with higher accuracy and to derive a more detailed stability diagram of superfluid droplets from the measurements. Furthermore, probing smaller droplet sizes will reveal possible finite size effects. Corresponding work on self-bound quantum droplets in mixed BECs may provide complementary information by studying more dilute systems, potentially with greater control over their rotational states $[39,40]$.
This work was supported by the NSF Grant No. DMR1701077 (A. F. V.), by the U.S. Department of Energy, Office of Basic Energy Sciences, (DOE, OBES) Chemical Sciences, Geosciences and Biosciences Division, through Contract No. DE-AC02-05CH11231 (C. Ba., C. A. S., J. M., B. W. T., O. G.) as well as No. DE-AC0206CH11357 (Y. K., C. Bo.), No. DE-AC02-76SF00515 (C. Bo.), Université Fédérale Toulouse-Midi-Pyrénées throughout the "Chaires d'Attractivité 2014" Programme IMDYNHE (M. B.), and FIS2017-87801-P (AEI/FEDER, UE) (M. B., M. P.). Portions of this research were carried out at the Linac Coherent Light Source, a national user facility operated by Stanford University on behalf of the U.S. DOE, OBES under beam-time grant LP05: Superfluids far from equilibrium. We are grateful to John Bozek, Justin Kwok, Curtis Jones, Ken Ferguson, Sebastian Schorb, and Martin Seifrid for providing assistance during some experiments described in this Letter.

\footnotetext{
*To whom correspondence should be addressed. francesco.ancilotto@pd.infn.it

${ }^{\dagger}$ To whom correspondence should be addressed. ogessner@lbl.gov
} 
To whom correspondence should be addressed.

vilesov@usc.edu.

[1] S. Chandrasekhar, The stability of a rotating liquid drop, Proc. R. Soc. London Ser. A 286, 1 (1965).

[2] S. Cohen, F. Plasil, and W. J. Swiatecki, Equilibrium configurations of rotating charged or gravitating liquid masses with surface-tension. 2, Ann. Phys. (N.Y.) 82, 557 (1974).

[3] R. A. Brown and L. E. Scriven, The shape and stability of rotating liquid-drops, Proc. R. Soc. London Ser. A 371, 331 (1980).

[4] S. L. Butler, M. R. Stauffer, G. Sinha, A. Lilly, and R. J. Spiteri, The shape distribution of splash-form tektites predicted by numerical simulations of rotating fluid drops, J. Fluid Mech. 667, 358 (2011).

[5] L. Pitaevskii and S. Stringari, Bose-Einstein Condensation and Superfluidity (Oxford University Press, Oxford, 2016), Vol. 164.

[6] A. L. Fetter, Rotating trapped Bose-Einstein condensates, Rev. Mod. Phys. 81, 647 (2009).

[7] L.F. Gomez et al., Shapes and vorticities of superfluid helium nanodroplets, Science 345, 906 (2014).

[8] C. Bernando, R. P. Tanyag, C. Jones, C. Bacellar, M. Bucher et al., Shapes of rotating superfluid helium nanodroplets, Phys. Rev. B 95, 064510 (2017).

[9] D. Rupp et al., Coherent diffractive imaging of single helium nanodroplets with a high harmonic generation source, Nat. Commun. 8, 493 (2017).

[10] B. Langbehn, K. Sander, Y. Ovcharenko, C. Peltz, A. Clark et al., Three-Dimensional Shapes of Spinning Helium Nanodroplets, Phys. Rev. Lett. 121, 255301 (2018).

[11] F. Ancilotto, M. Barranco, and M. Pi, Spinning superfluid He-4 nanodroplets, Phys. Rev. B 97, 184515 (2018).

[12] D. L. Whitaker, M. A. Weilert, C. L. Vicente, H. J. Maris, and G. M. Seidel, Oscillations of charged helium II drops, J. Low Temp. Phys. 110, 173 (1998).

[13] L. Childress, M. P. Schmidt, A. D. Kashkanova, C. D. Brown, G. I. Harris, A. Aiello, F. Marquardt, and J. G. E. Harris, Cavity optomechanics in a levitated helium drop, Phys. Rev. A 96, 063842 (2017).

[14] A. L. Fetter, Vortex nucleation in deformed rotating cylinders, J. Low Temp. Phys. 16, 533 (1974).

[15] M. O. Oktel, Vortex lattice of a Bose-Einstein condensate in a rotating anisotropic trap, Phys. Rev. A 69, 023618 (2004).

[16] S. Sinha and G. V. Shlyapnikov, Two-Dimensional BoseEinstein Condensate Under Extreme Rotation, Phys. Rev. Lett. 94, 150401 (2005).

[17] P. Sanchez-Lotero and J. J. Palacios, Vortices in a rotating Bose-Einstein condensate under extreme elongation, Phys. Rev. A 72, 043613 (2005).

[18] N. Lo Gullo, T. Busch, and M. Paternostro, Structural change of vortex patterns in anisotropic Bose-Einstein condensates, Phys. Rev. A 83, 053612 (2011).

[19] K. Deconde and R. E. Packard, Measurement of Equilibrium Critical Velocities for Vortex Formation in SuperfluidHelium, Phys. Rev. Lett. 35, 732 (1975).

[20] F. Ancilotto, M. Pi, and M. Barranco, Vortex arrays in nanoscopic superfluid helium droplets, Phys. Rev. B 91, 100503(R) (2015).
[21] J. R. Abo-Shaeer, C. Raman, J. M. Vogels, and W. Ketterle, Observation of vortex lattices in Bose-Einstein condensates, Science 292, 476 (2001).

[22] L. Struder et al., Large-format, high-speed, x-ray pnCCDs combined with electron and ion imaging spectrometers in a multipurpose chamber for experiments at 4th generation light sources, Nucl. Instrum. Methods Phys. Res., Sect. A 614, 483 (2010).

[23] K. R. Ferguson et al., The atomic, molecular and optical science instrument at the linac coherent light source, J. Synchrotron Radiat. 22, 492 (2015).

[24] L. F. Gomez, E. Loginov, R. Sliter, and A. F. Vilesov, Sizes of large He droplets, J. Chem. Phys. 135, 154201 (2011).

[25] R. M. P. Tanyag, C. F. Jones, C. Bernando, D. Verma, S. M. O. O'Connell, and A.F. Vilesov, in Cold Chemistry: Molecular Scattering and Reactivity Near Absolute Zero, edited by A. Osterwalder and O. Dulieu (Royal Society of Chemistry, Cambridge, 2018), p. 389.

[26] M. Hartmann, R. E. Miller, J. P. Toennies, and A. Vilesov, Rotationally Resolved Spectroscopy of $\mathrm{SF}_{6}$ in LiquidHelium Clusters - a Molecular Probe of Cluster Temperature, Phys. Rev. Lett. 75, 1566 (1995).

[27] C. F. Jones, C. Bernando, R. P. Tanyag, C. Bacellar, K. Ferguson et al., Coupled motion of Xe clusters and quantum vortices in He nanodroplets, Phys. Rev. B 93, 180510(R) (2016).

[28] O. Gessner and A. F. Vilesov, Imaging quantum vortices in superfluid helium Droplets, Annu. Rev. Phys. Chem. 70, 173 (2019).

[29] F. Dalfovo, R. Mayol, M. Pi, and M. Barranco, Pinning of Quantized Vortices in Helium Drops by Dopant Atoms and Molecules, Phys. Rev. Lett. 85, 1028 (2000).

[30] G. P. Bewley, D. P. Lathrop, and K. R. Sreenivasan, Superfluid helium-Visualization of quantized vortices, Nature (London) 441, 588 (2006).

[31] R. M. P. Tanyag et al., Communication: X-ray coherent diffractive imaging by immersion in nanodroplets, Struct. Dyn. 2, 051102 (2015).

[32] See Supplemental Materials at http://link.aps.org/ supplemental/10.1103/PhysRevLett.124.215301 for additional information on the determination of the droplet parameters, some deviations between the classical and superfluid droplet shapes and details of DFT calculations, which includes Refs. [7,8,11,27,33-37].

[33] S. T. Nam, G. H. Bauer, and R. J. Donnelly, Vortex patterns in a freely rotating superfluid, J. Korean Phys. Soc. 29, 755 (1996).

[34] R. P. Feynman, in Chapter II Application of Quantum Mechanics to Liquid Helium, edited by C. J. Gorter (North-Holland Publishing Company, Amsterdam, 1955), Vol. 2, p. 17.

[35] F. Coppens, F. Ancilotto, M. Barranco, N. Halberstadt, and M. $\mathrm{Pi}$, Capture of $\mathrm{Xe}$ and $\mathrm{Ar}$ atoms by quantized vortices in He-4 nanodroplets, Phys. Chem. Chem. Phys. 19, 24805 (2017).

[36] F. Ancilotto, M. Barranco, F. Coppens, J. Eloranta, N. Halberstadt, A. Hernando, D. Mateo, and M. Pi, Density functional theory of doped superfluid liquid 
helium and nanodroplets, Int. Rev. Phys. Chem. 36, 621 (2017).

[37] F. Ancilotto, M. Pi, and M. Barranco, Vortex arrays in a rotating superfluid He-4 nanocylinder, Phys. Rev. B 90, 174512 (2014).

[38] C. Bernando and A. F. Vilesov, Kinematics of the doped quantum vortices in superfluid helium droplets, J. Low Temp. Phys. 191, 242 (2018).
[39] C. R. Cabrera, L. Tanzi, J. Sanz, B. Naylor, P. Thomas, P. Cheiney, and L. Tarruell, Quantum liquid droplets in a mixture of Bose-Einstein condensates, Science 359, 301 (2018).

[40] H. Kadau, M. Schmitt, M. Wenzel, C. Wink, T. Maier, I. Ferrier-Barbut, and T. Pfau, Observing the Rosensweig instability of a quantum ferrofluid, Nature (London) 530, 194 (2016). 